Article

\title{
Highly Sensitive Label-Free Detection of Small Molecules with an Optofluidic Microbubble Resonator
}

\author{
Zihao $\mathrm{Li}^{\dagger}$, Chenggang $\mathrm{Zhu}^{\dagger}{ }^{\dagger}$, Zhihe Guo, Bowen Wang, Xiang $\mathrm{Wu}{ }^{*}$ and Yiyan Fei * \\ Department of Optical Science and Engineering, Shanghai Engineering Research Center of Ultra-Precision \\ Optical Manufacturing, Key Laboratory of Micro and Nano Photonic Structures (Ministry of Education), \\ Fudan University, Shanghai 200433, China; 15210720012@fudan.edu.cn (Z.L.); 16110720026@fudan.edu.cn (C.Z.); \\ 17110720004@fudan.edu.cn (Z.G.); 16210720012@fudan.edu.cn (B.W.) \\ * Correspondence: wuxiang@fudan.edu.cn (X.W.); fyy@fudan.edu.cn (Y.F.); Tel.: +86-021-6564-2092 (Y.F.) \\ + These authors contributed equally to this work.
}

Received: 8 April 2018; Accepted: 29 May 2018; Published: 31 May 2018

\begin{abstract}
The detection of small molecules has increasingly attracted the attention of researchers because of its important physiological function. In this manuscript, we propose a novel optical sensor which uses an optofluidic microbubble resonator (OFMBR) for the highly sensitive detection of small molecules. This paper demonstrates the binding of the small molecule biotin to surface-immobilized streptavidin with a detection limit reduced to $0.41 \mathrm{pM}$. Furthermore, binding specificity of four additional small molecules to surface-immobilized streptavidin is shown. A label-free OFMBR-based optical sensor has great potential in small molecule detection and drug screening because of its high sensitivity, low detection limit, and minimal sample consumption.
\end{abstract}

Keywords: label-free sensor; optofluidic microbubble resonator; detection of small molecules

\section{Introduction}

Methods to detect small molecular analytes $(<1000 \mathrm{Da})$ have found several, important uses in the bio-medical, food, and environmental fields [1-3]. In biomedical analyses, several small molecules such as steroids, thyroid hormones, and peptides derived from disease-specific proteins have been utilized as diagnostic markers [4,5]. Additionally, the detection of small molecules analytes could serve a vital role in the identification of bacterial pathogens in infectious diseases [6,7].

To detect small molecules, several electrical, mechanical, and optical sensors have been developed, such as the nanomechanical resonator sensor, nanowire sensor, and surface plasmon resonance sensor. Among them, optical detection methods stand out because of their advantages, such as greater sensitivity, electrical passiveness, and robustness. To date, the most widely used optical detection technology uses labels, such as radio- or fluorescent-labeling, for detection. Although popular and useful, the labeling step requires additional time and cost, and even worse, a labeled approach can significantly alter the activities of small molecules and lead to inaccurate conclusions. Various label-free optical techniques, such as surface plasmon resonance (SPR) [8], resonant waveguide grating (RWG) [9], resonant mirror (RM) [10,11], and high-Q optical microcavities [12-16] have been developed, but their sensitivities diminish with the size of the molecule, making it extremely challenging to detect small molecules. To date, the detection limit for small molecules with label-free optical detection technologies are in the range of $\mu \mathrm{M}$ to $\mathrm{nM}[17,18]$.

An optofluidic microbubble resonator (OFMBR) that supports high-Q whispering gallery modes (WGMs) is a promising candidate for the development of sensors [19-23] because of its unique hollow-core structure, capabilities of integration with microfluidics, high sensitivity, and excellent 
optical confinement of the WGMs [24,25]. Light couples into the microbubble resonator through the fiber taper and the WGMs shift in response to the change of the refractive index in the medium surrounding the resonator, which leads to additional applications of OFMBR in label-free biosensing. However, the OFMBR is typically exposed in the air, which is susceptible to environmental changes, greatly diminishing its performance capabilities in small molecule detection.

In this paper, we propose a packaged OFMBR sensor by fixing the ends of a microbubble and fiber taper on glass substrate with glue, and sealing the OFMBR inside a glass box. By immobilization of streptavidin on the inner surface of microbubble and injection of various concentrations of small molecule biotin, we obtained real-time binding curves of biotin to immobilized streptavidin, which gave us a binding affinity of $6.7 \times 10^{14} \mathrm{M}^{-1}$. The detection limit of biotin to immobilized streptavidin on the packaged OFMBR sensor was determined to be $0.41 \mathrm{pM}$. In addition, we flew four small molecules over a streptavidin-immobilized OFMBR sensor; only magnolol bound to the surface-immobilized streptavidin, with binding affinity of $4.7 \times 10^{10} \mathrm{M}^{-1}$. The packaged OFMBR sensor was demonstrated to be able to detect small molecules with high sensitivity and low detection limit, which has significant application potential in small molecule detection and drug screening.

\section{Materials and Method}

\subsection{Materials}

The 3-glycidoxypropyltrimethoxysilan (GOPTS) was from Sinopharm Chemical Regent Company (Shanghai, China). Bovine serum albumin (BSA) and phosphate buffered saline (PBS) were from Sigma-Aldrich (St. Louis, MO, USA). Streptavidin (SA) was from Life Technologies (Shanghai, China). Biotin was from Aladdin Industrial Corporation (Shanghai, China). Magnolol, medetomidine $\mathrm{HCl}$, cetrimonium bromide, and reboxetine mesylat were from Selleck (Houston, TX, USA). The low refractive index polymer MY133 was from MY Polymers (Ness Ziona, Israel). The UV glue was from Thorlabs (Shanghai, China).

\subsection{The Packaged OFMBR Sensor for Biomolecular Interaction Detection}

Figure 1a shows the sketch of the OFMBR detection system, which consists of a tunable, continuous diode laser, with a tuning range from $765 \mathrm{~nm}$ to $781 \mathrm{~nm}$ (TLB6700, New Focus, San Diego, CA, USA), a polarization controller (EPC300, Connet Fiber Optics, Shanghai, China), a packaged OFMBR sensor, a photon detector (DET10C, Thorlabs, Newton, NJ, USA), and an oscilloscope at a working sampling rate of 500,000 S/s (TDS3012, Tektronix, Portland, OR, USA). Figure $1 \mathrm{~b}$ shows the structure of a packaged OFMBR sensor. The OFMBR was on a glass substrate, with wall thickness about $2-4 \mu \mathrm{m}$, lying perpendicular to a fiber taper; optimal coupling was achieved by adjusting the gap between them. To fix the position and wrap the fiber, low refractive index polymer MY133 was on both ends of the fiber taper. To fix the OFMBR on the substrate, UV glue (NOA68, Thorlabs, Newton, NJ, USA) covered both ends of the microbubble. In addition, a cover glass was placed on top of the four piles on the glass substrate corners to seal the packaged sensor. The packaged OFMBR sensor was solidified in the air by its exposure to a UV lamp at $365 \mathrm{~nm}$ for $5 \mathrm{~min}$. Instead of putting MY133 around the coupling region of OFMBR with fiber taper and on the ends of the fiber taper as described before [25], we put polymer MY133 only on the ends of the fiber taper in such a way that excited WGMs were not affected; as a result, the as-packaged OFMBR sensor was more sensitive than that described before [25]. 

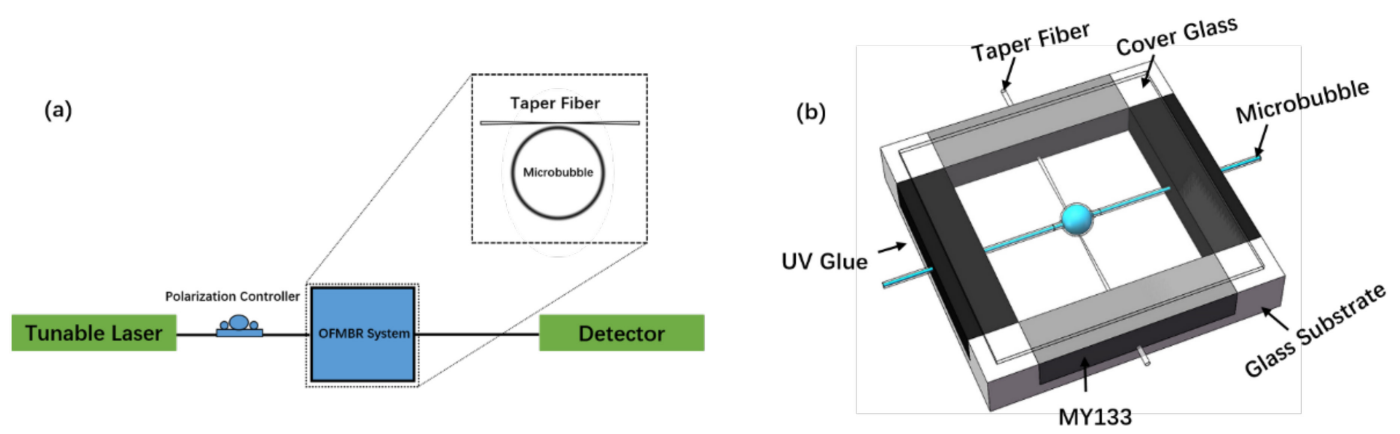

Figure 1. (a) Sketch of the detection system for the OFMBR sensor; (b) Structure of the packaged OFMBR sensor.

When biological molecules aggregated at the inner surface of the microbubble, they interacted with the evanescent part of the WGM field. Subsequently, a wavelength shift occurred as a result of the change of the radius or the refractive index of the microbubble. The resonance wavelength shift $\Delta_{\lambda}$ was proportional to the surface density of the small molecules $\mathrm{N}_{\mathrm{AB}}(\mathrm{t})$, which is expressed as [26],

$$
\Delta_{\lambda}=\alpha_{\mathrm{ex}} \lambda \mathrm{S} \frac{2 \pi \sqrt{\mathrm{n}_{2}^{2}-\mathrm{n}_{3}^{2}}}{\varepsilon_{0} \lambda^{2}} \frac{\mathrm{n}_{2}}{\mathrm{n}_{3}^{2}} \times \mathrm{N}_{\mathrm{AB}}(\mathrm{t})=\mu \times \mathrm{N}_{\mathrm{AB}}(\mathrm{t})
$$

$\mathrm{n}_{2}, \mathrm{n}_{3}$ are the refractive index values of the OFMBR's wall (quartz) and the OFMBR's core (air or liquid), $\alpha_{\mathrm{ex}}$ is the excess polarizability of the biomolecules, $S$ is the sensitivity of refractive index. By following the wavelength shift variations with time, binding kinetics of biomolecule to the OFMBR inner surface was revealed.

\subsection{Detection of Small Molecule Binding to Surface-Immobilized Streptavidin}

Figure 2a schematically shows the functionalization of the inner surface of the microbubble with epoxy groups by using 3-glycidoxypropyltrimethoxysilan (GOPTS, Sinopharm Chemical Regent Company, Shanghai, China). The inner surface of OFMBR was first activated by $12 \% \mathrm{NaOH}$ (Lingfeng Chemical Regent, Shanghai, China) for $1 \mathrm{~h}$ to obtain $\mathrm{OH}$ terminal groups. The microbubble was then filled with 1\% GOPTS in 95\% ethanol for $2 \mathrm{~h}$, followed by washing with toluene, ethanol, and deionized water for $10 \mathrm{~min}$ each to remove residual unreacted GOPTS. The microbubble was finally dried in an oven at $120{ }^{\circ} \mathrm{C}$ for $2 \mathrm{~h}$. The functionalized microbubble surface, having epoxy groups, formed covalent linkages with amine groups on proteins which were thus immobilized on the inner surface of microbubble with high efficiency.

Figure $2 \mathrm{~b}$ shows experimental protocols for the detection of small molecule binding to surface-immobilized streptavidin. Protein streptavidin was first immobilized on the inner surface of the OFMBR by its incubation with streptavidin, at a concentration of $7.7 \mathrm{uM}$ for $2 \mathrm{~h}$, followed by $1 \times$ PBS washing for $0.5 \mathrm{~h}$. The inner surface was then blocked with $7.6 \mathrm{uM}$ BSA at a flow rate of $2.5 \mu \mathrm{L} / \mathrm{min}$ for $2 \mathrm{~h}$ and followed by washing with $1 \times \mathrm{PBS}$ for $0.5 \mathrm{~h}$.

Each binding curve of small molecule to surface-immobilized streptavidin included baseline, association phase, and dissociation phase. During baseline, $1 \times$ PBS flew over surface-immobilized streptavidin at a flow rate of $2 \mu \mathrm{L} / \mathrm{min}$ for $6 \mathrm{~min}$. During the association phase, small molecule solution flew over surface-immobilized streptavidin for $22 \mathrm{~min}$ at a flow rate of $2 \mu \mathrm{L} / \mathrm{min}$. Dissociation phase included $1 \times$ PBS flowing over the OFMBR at a flow rate of $2 \mu \mathrm{L} / \mathrm{min}$ for $8 \mathrm{~min}$. Specific binding of small molecule to surface-immobilized streptavidin caused red-shift of the OFMBR WGMs, which was recorded from the beginning of the baseline to the end of the dissociation phase at a time resolution of $1 \mathrm{~s}$. All binding curves were normalized against respective maximal resonance wavelength shift which does not affect reaction kinetic rate constants. 
(a)

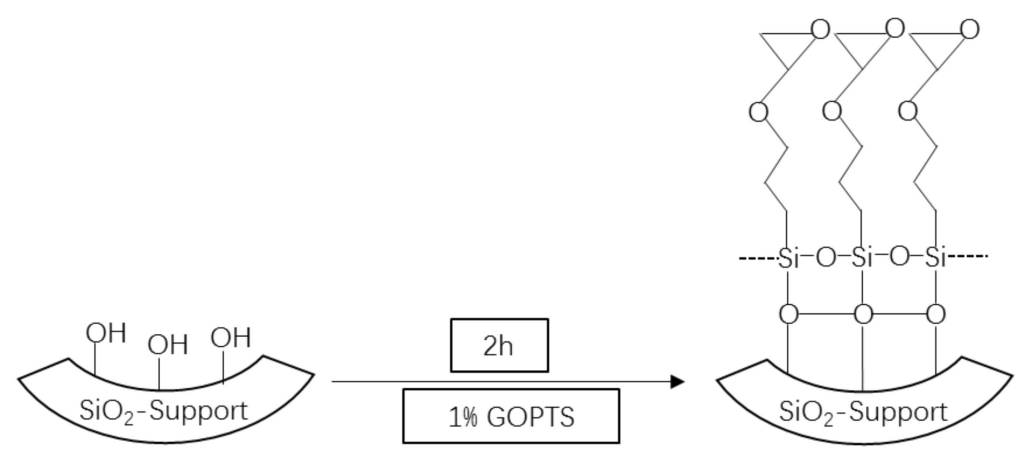

(b)

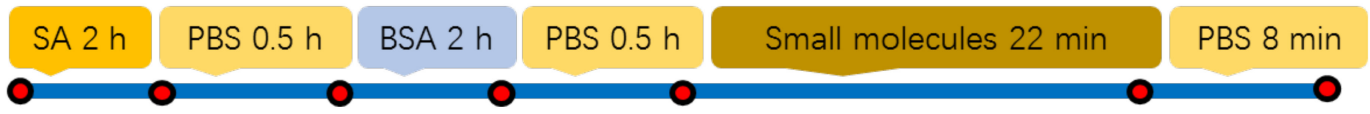

Figure 2. (a) Schematic illustration of surface functionalization of microbubble surface with epoxy groups; (b) Flow diagram of experimental processes for small biomolecule binding to surface-immobilized streptavidin.

\section{Results}

\subsection{Transmission Spectra and Q Factors of OFMBR Sensor}

An OFMBR sensor consists of a microbubble coupled with a fiber taper. The OFMBR sensor exhibits high sensitivity due to the presence of a significant part of the WGM field close to the inner surface of the microbubble. In addition, the OFMBR sensor has excellent fluidic capability because of its intrinsic hollow structure. However, the exposed OFMBR sensor is susceptible to environmental changes and usually an exposed sensor lasts less than $12 \mathrm{~h}$. We thus propose to package the OFMBR sensor within a sealed glass box (as described in Section 2.2) to reduce the impact of the environment.

Figure $3 \mathrm{a}, \mathrm{b}$ show transmission spectra of an OFMBR sensor with air inside before packaging and $24 \mathrm{~h}$ after packaging within a wavelength range of 770-780 nm, respectively. After packaging, WGMs remained excited. The decreased intensity of the transmission light after packaging resulted from the loss of scattering and absorption due to the coating of MY133 around the ends of the fiber taper. Figure $3 c, d$ show fine scan of the transmission spectra of OFMBR sensor before and after packaging around $774.3 \mathrm{~nm}$. Both spectra show the same four WGMs, indicating that the excited WGMs were all reserved after the packaging process. The typical $Q$ factors were obtained by Lorenz fits of the WGMs, indicated by the red curves in Figure $3 c, d$ and the $Q$ values before and after packaging were approximately $3.7 \times 10^{5}$ and $3.0 \times 10^{5}$, respectively. The $Q$ values before and after packaging were on the same order of magnitude, proving good maintenance of $Q$ values after the packaging. The slight decrease of $Q$ values was attributable to changes of coupling coefficiency between the microbubble and the fiber taper, caused by a slight movement of taper fiber during the packaging process. Figure $3 \mathrm{e}, \mathrm{f}$ show resonant wavelength shift varying with time of an OFMBR sensor before and after packaging. The standard deviation of resonant wavelength shifts decreased from $2.6 \mathrm{pm}$ to $0.15 \mathrm{pm}$ after packaging, indicating that sensor packaging greatly decreased resonant wavelength drift. In addition, performance of the as-packaged OFMBR sensor was stable for months. Results indicate that OFMBR sensor packaging could provide lower noise and longer lift time, without sacrificing its performance. 

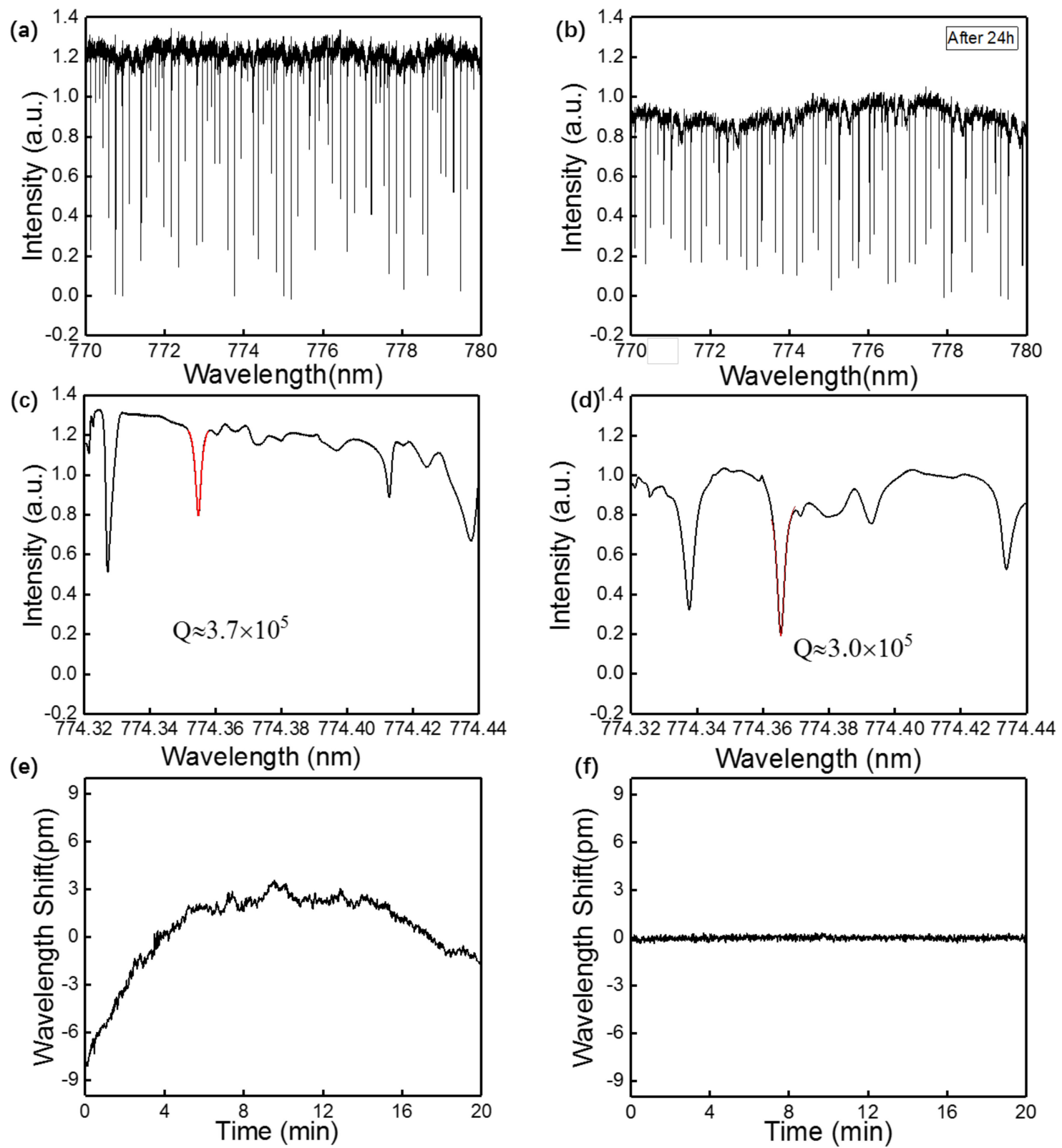

Figure 3. (a) Transmission spectra of an OFMBR before packaging in the wavelength range between $770 \mathrm{~nm}$ and $780 \mathrm{~nm}$; (b) Transmission spectra of an OFMBR $24 \mathrm{~h}$ after packaging in the wavelength range between $770 \mathrm{~nm}$ and $780 \mathrm{~nm}$; (c) Fine scan of the transmission spectra before packaging around $774.3 \mathrm{~nm}$; (d) Fine scan of the transmission spectra after packaging around $774.3 \mathrm{~nm}$; (e) Resonant wavelength shift of an OFMBR sensor varying with time before packaging; (f) Resonant wavelength shift of an OFMBR sensor varying with time after packaging.

\subsection{Binding Kinetics of Biotin to Surface-Immobilized Streptavidin}

Biotin is a small molecule with molecular weight of $224 \mathrm{Da}$, which binds strongly to streptavidin. We used biotin binding to surface-immobilized streptavidin as a model system to study the performance of a packaged OFMBR sensor on small molecule detection. We measured binding kinetics of biotin to surface-immobilized streptavidin at respective biotin concentrations of $205 \mathrm{pM}$, $410 \mathrm{pM}$, and $820 \mathrm{pM}$ on three fresh OFMBR sensors; Figure 4a shows the three normalized real-time binding curves among them. Vertical lines indicate the start of the association and dissociation phases. Real-time binding curves contain information on kinetic rate constants, $\mathrm{k}_{\text {on }}$ (association rate) and 
$\mathrm{k}_{\text {off }}$ (dissociation rate). For the monophasic molecular interaction with stoichiometry 1:1, the affinity constant $\mathrm{k}_{\mathrm{a}}$ is the ratio of $\mathrm{k}_{\text {on }}$ to $\mathrm{k}_{\text {off }}$

$$
\begin{gathered}
\mathrm{A}+\mathrm{B} \stackrel{\mathrm{k}_{\text {on }}}{\longrightarrow} \mathrm{AB} \\
\mathrm{AB} \stackrel{\mathrm{k}_{\text {off }}}{\longrightarrow} \mathrm{A}+\mathrm{B} \\
\mathrm{k}_{\mathrm{a}}=\frac{\mathrm{k}_{\text {on }}}{\mathrm{k}_{\text {off }}}
\end{gathered}
$$

To extract binding kinetic rate constants, we used a Langmuir reaction model. As shown in Equations (5), small molecules are assumed to bind to surface-immobilized streptavidin at a rate proportional to the association rate $\mathrm{k}_{\mathrm{on}}$, small molecule concentration $[\mathrm{A}]$, and the unbonded binding sites $\mathrm{N}_{[\mathrm{AB}]_{\max }}-\mathrm{N}_{[\mathrm{AB}]}$ at time less than $\mathrm{t}_{0}$. After the small molecule solution was replaced with a PBS buffer at time $\mathrm{t}_{0}$, small molecule-protein complexes dissociated at a rate proportional to dissociation rate $\mathrm{k}_{\text {off }}$ and number of complexes per sensing area $\mathrm{N}_{[\mathrm{AB}]}$.

$$
\left\{\begin{array}{c}
\frac{\mathrm{dN}_{[\mathrm{AB}]}}{\mathrm{dt}}=\mathrm{k}_{\mathrm{on}}[\mathrm{A}]\left(\mathrm{N}_{[\mathrm{AB}]_{\max }}-\mathrm{N}_{[\mathrm{AB}]}\right)-\mathrm{k}_{\text {off }} \mathrm{N}_{[\mathrm{AB}]}, \mathrm{t} \leq \mathrm{t}_{0}, \\
\frac{\mathrm{dN_{[AB] }}}{\mathrm{dt}}=-\mathrm{k}_{\mathrm{off}} \mathrm{N}_{[\mathrm{AB}]}, \mathrm{t}>\mathrm{t}_{0}
\end{array}\right.
$$

$\mathrm{N}_{[\mathrm{AB}]_{\max }}$ is the maximal number of binding sites per sensing area. The number of small molecule complexes per unit sensing area $\mathrm{N}_{[\mathrm{AB}]}$ is calculated to be,

$$
\left\{\begin{array}{c}
\mathrm{N}_{\mathrm{AB}}(\mathrm{t})=\frac{\mathrm{k}_{\text {on }}[\mathrm{A}] \mathrm{N}_{[\mathrm{AB}]_{\max }}}{\mathrm{k}_{\text {on }}[\mathrm{A}]+\mathrm{k}_{\text {off }}}\left(1-\mathrm{e}^{-\left(\mathrm{k}_{\text {on }}[\mathrm{A}]+\mathrm{k}_{\text {off }}\right) t}\right), t \leq \mathrm{t}_{0}, \\
\mathrm{~N}_{\mathrm{AB}}(\mathrm{t})=\frac{\mathrm{k}_{\text {on }}[\mathrm{A}] \mathrm{N}_{[\mathrm{AB}] \max }}{\mathrm{k}_{\text {on }}[\mathrm{A}]+\mathrm{k}_{\text {off }}}\left(1-\mathrm{e}^{-\left(\mathrm{k}_{\text {on }}[\mathrm{A}]+\mathrm{k}_{\text {off }}\right) \mathrm{t}_{0}}\right) \mathrm{e}^{-\mathrm{k}_{\text {off }}\left(\mathrm{t}-\mathrm{t}_{0}\right)}, t>\mathrm{t}_{0}
\end{array}\right.
$$

We obtained reaction kinetic rate constants $k_{\text {on }}$ and $k_{\text {off }}$ by globally fitting three normalized binding curves in Figure 4a with Equations (6). As shown in Table 1, the binding affinity between small molecule biotin and surface-immobilized streptavidin was $6.7 \times 10^{14} \mathrm{M}^{-1}$, which is close to the affinity range $\left(10^{13}-10^{14} \mathrm{M}^{-1}\right)$ reported by others $[27,28]$. Figure $4 \mathrm{~b}$ shows the binding curve of surface-immobilized streptavidin to flowing biotin at a concentration of $0.41 \mathrm{pM}$, which is significantly lower than the detection limit achieved on other label-free optical sensing systems [12].
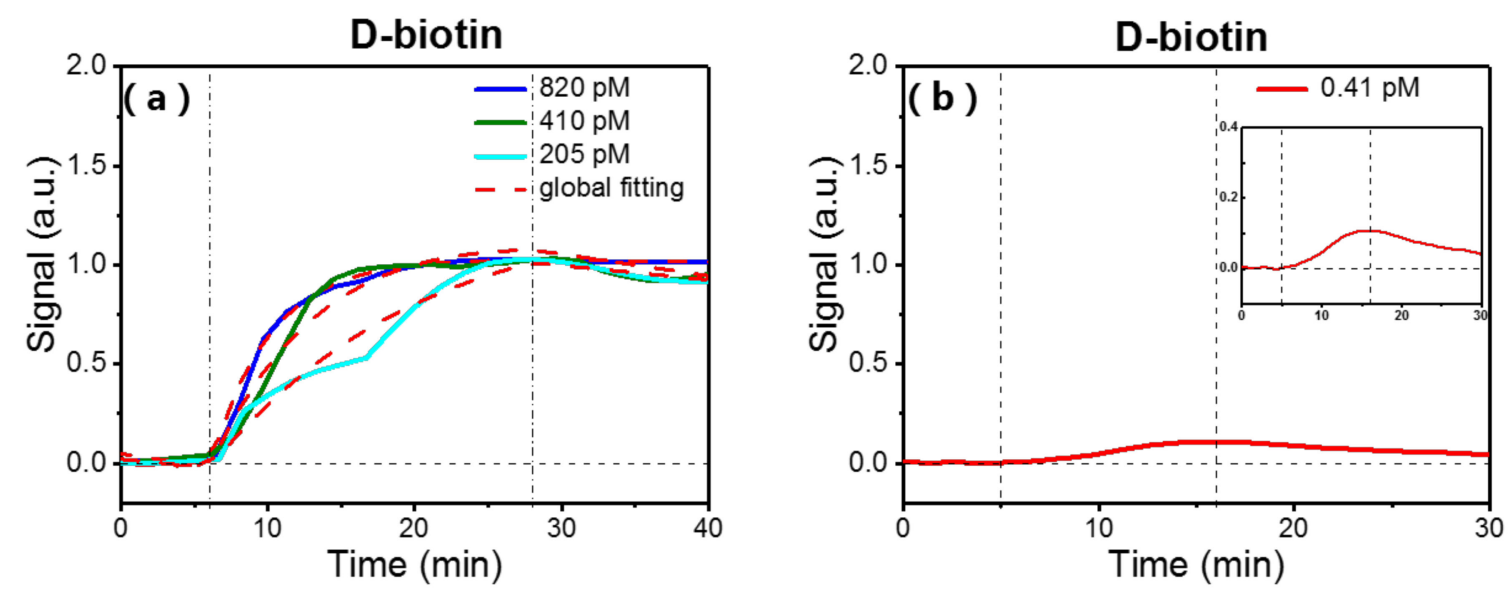

Figure 4. (a) Binding curves of surface-immobilized streptavidin with flowing biotin at respective concentrations of $205 \mathrm{pM}, 410 \mathrm{pM}$, and $820 \mathrm{pM}$. Vertical lines indicate start of association and dissociation phases. Red dashed lines are global fitting results with the Langmuir reaction model; (b) Specific binding curve of surface-immobilized streptavidin with biotin at a concentration of $0.41 \mathrm{pM}$. Inset shows enlarged view of the binding curve. 
Table 1. Kinetic constants of biotin and magnolol binding to immobilized streptavidin.

\begin{tabular}{cccc}
\hline Small Molecule & $\mathbf{k}_{\mathbf{o n}}(\mathbf{m i n} \mathbf{n M})^{\mathbf{- 1}}$ & $\mathbf{k}_{\mathbf{o f f}}(\mathbf{m i n})^{-\mathbf{1}}$ & $\left.\mathbf{k}_{\mathbf{a}} \mathbf{( M}\right)^{-\mathbf{1}}$ \\
\hline Biotin & 0.30 & $4.5 \times 10^{-7}$ & $6.7 \times 10^{14}$ \\
Magnolol & 0.29 & $6.2 \times 10^{-3}$ & $4.7 \times 10^{10}$ \\
\hline
\end{tabular}

\subsection{Specificity of Small Molecules Binding to Surface-Immobilized Streptavidin}

To study binding specificity of small molecules to surface-immobilized streptavidin on the packaged OFMBR sensor, we flew four small molecules at respective concentrations of $205 \mathrm{pM}$ sequentially over the surface-immobilized streptavidin. Three small molecules, medetomidine $\mathrm{HCl}$, cetrimonium bromided, and reboxetine mesylat were randomly selected from our compound library. Magnolol, a binding ligand of streptavidin screened from 3375 compounds [29], was also included. Figure 5 show that the three randomly selected small molecules did not bind to the surface-immobilized streptavidin and magnolol bound specifically to streptavidin, indicating that the as-packaged OFMBR sensor functionalized with streptavidin binds specifically with small molecules. From binding curves between streptavidin and magnolol at concentrations of 100 pM, 500 pM, and 1000 pM, the binding affinity between them was found to be $4.7 \times 10^{10} \mathrm{M}^{-1}$.
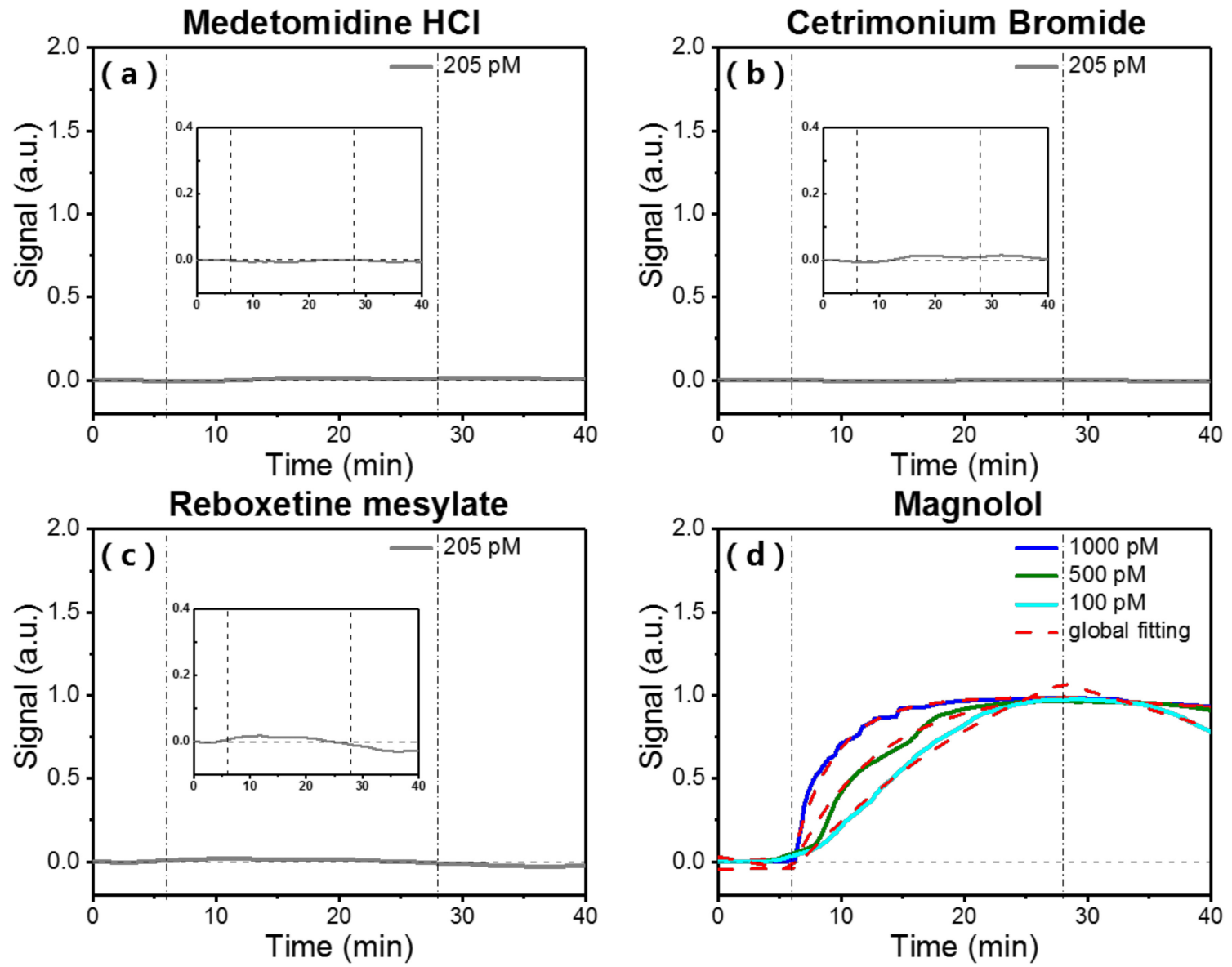

Figure 5. Real-time binding curves of small molecules (a) medetomidine $\mathrm{HCl}$; (b) cetrimonium bromide; (c) reboxetine mesylate with surface-immobilized streptavidin on packaged OFMBR sensor at respective concentration of $205 \mathrm{pM}$ (Insets show enlarged views of the binding curves); (d) Binding curves of surface-immobilized streptavidin with flowing magnolol at concentrations of $100 \mathrm{pM}, 500 \mathrm{pM}$, and $1000 \mathrm{pM}$. Vertical lines are the starts of association and dissociation phases. 


\section{Discussion and Conclusions}

The OFMBR sensor was demonstrated to be capable of detecting biomolecular interactions with high sensitivity, based on WGMs exited through a coupling of a microbubble resonator (OFMBR) and a fiber taper. Traditionally, a OFMBR sensor is exposed to the air and the sensor's performance is susceptible to environmental changes, especially dirt in the air. In this way, the exposed OFMBR sensor usually lasts less than $12 \mathrm{~h}$. In addition, it is not convenient to carry the exposed OFMBR sensor. To improve the stability of an OFMBR sensor, we fixed the ends of the sensor with glue on the glass substrate and put the packaged sensor inside a glass box. The as-packaged OFMBR sensor displayed good stability, lasting for months. A significant difference between the package protocols of this work and the package protocols described before [25] is that we did not wrap the coupling position between the microbubble and the fiber taper with MY133; accordingly, the high radial order modes were reserved to provide high sensitivity.

Detection limit of the packaged OFMBR sensor can be further optimized by enhancing sensor sensitivity, increasing surface density of immobilized protein ligand, and reducing noise level. Thin shells and high radial modes are beneficial for improving sensor sensitivity. Optimization of surface functionalization and surface immobilization protocols is expected to maximize protein surface density. Noise level can be reduced by increasing the stability of the OFMBR sensor, keeping it at a constant temperature, and using a self-referencing sensing scheme, such as mode-splitting method [14].

In this paper, we demonstrate a novel optical sensor for label-free small molecule detection with low detection limit. With surface-immobilized streptavidin on the inner surface of a packaged OFMBR sensor, binding of small molecule biotin to streptavidin can be detected with a reduced detection limit at $0.41 \mathrm{pM}$. The specificity of small molecule binding on OFMBR sensor was demonstrated with four additional small molecules. Such a sensitive optical sensor will find wide applications in medical diagnoses, treatment of diseases, and drug screening.

Author Contributions: Z.L., Z.G., B.W., and X.W. designed and performed the experiments; C.Z. contributed samples and analyzed experimental data; Z.L. and Y.F. wrote and revised the paper.

Acknowledgments: This work was supported by the Special Project of National Key R\&D Program of the Ministry of Science and Technology of China (2106YFC0201401), the National Natural Science Foundation of China (NSFC) $(61505032,61378080,61327008)$.

Conflicts of Interest: The authors declare no conflicts of interest.

\section{References}

1. Zhang, J.; Yang, P.L.; Gray, N.S. Targeting cancer with small molecule kinase inhibitors. Nat. Rev. Cancer 2009, 9, 28. [CrossRef] [PubMed]

2. Wang, W.U.; Chen, C.; Lin, K.H.; Fang, Y.; Lieber, C.M. Label-free detection of small-molecule-Protein interactions by using nanowire nanosensors. Proc. Natl. Acad. Sci. USA 2005, 102, 3208-3212. [CrossRef] [PubMed]

3. Strausberg, R.L.; Schreiber, S.L. From knowing to controlling: A path from genomics to drugs using small molecule probes. Science 2003, 300, 294-295. [CrossRef] [PubMed]

4. Kenakin, T.; Christopoulos, A. Signalling bias in new drug discovery: Detection, quantification and therapeutic impact. Nat. Rev. Drug Discov. 2013, 12, 205. [CrossRef] [PubMed]

5. Holliger, P.; Hudson, P.J. Engineered antibody fragments and the rise of single domains. Nat. Biotechnol. 2005, 23, 1126. [CrossRef] [PubMed]

6. Gooding, J.J. Biosensor technology for detecting biological warfare agents: Recent progress and future trends. Anal. Chim. Acta 2006, 559, 137-151. [CrossRef]

7. Fitch, J.P.; Raber, E.; Imbro, D.R. Technology challenges in responding to biological or chemical attacks in the civilian sector. Science 2003, 302, 1350-1354. [CrossRef] [PubMed]

8. Anker, J.N.; Hall, W.P.; Lyandres, O.; Shah, N.C.; Zhao, J.; Van Duyne, R.P. Biosensing with plasmonic nanosensors. Nat. Mater. 2008, 7, 442-453. [CrossRef] [PubMed] 
9. Fang, Y.; Ferrie, A.M.; Fontaine, N.H.; Mauro, J.; Balakrishnan, J. Resonant waveguide grating biosensor for living cell sensing. Biophys. J. 2006, 91, 1925-1940. [CrossRef] [PubMed]

10. Owen, V. Real-time optical immunosensors-A commercial reality. Biosens. Bioelectron. 1997, 12, i-ii. [CrossRef]

11. Daghestani, H.N.; Day, B.W. Theory and applications of surface plasmon resonance, resonant mirror, resonant waveguide grating, and dual polarization interferometry biosensors. Sensors 2010, 10, 9630-9646. [CrossRef] [PubMed]

12. Vollmer, F.; Yang, L. Review Label-free detection with high-Q microcavities: A review of biosensing mechanisms for integrated devices. Nanophotonics 2012, 1, 267-291. [CrossRef] [PubMed]

13. Soria, S.; Berneschi, S.; Brenci, M.; Cosi, F.; Nunzi Conti, G.; Pelli, S.; Righini, G.C. Optical microspherical resonators for biomedical sensing. Sensors 2011, 11, 785-805. [CrossRef] [PubMed]

14. Li, M.; Wu, X.; Liu, L.; Fan, X.; Xu, L. Self-referencing optofluidic ring resonator sensor for highly sensitive biomolecular detection. Anal. Chem. 2013, 85, 9328-9332. [CrossRef] [PubMed]

15. Zhang, X.; Liu, L.; Xu, L. Ultralow sensing limit in optofluidic micro-bottle resonator biosensor by self-referenced differential-mode detection scheme. Appl. Phys. Lett. 2014, 104, 033703. [CrossRef]

16. Ren, L.; Wu, X.; Li, M.; Zhang, X.; Liu, L.; Xu, L. Ultrasensitive label-free coupled optofluidic ring laser sensor. Opt. Lett. 2012, 37, 3873-3875. [CrossRef] [PubMed]

17. Arlett, J.L.; Myers, E.B.; Roukes, M.L. Comparative advantages of mechanical biosensors. Nat. Nanotechnol. 2011, 6, 203. [CrossRef] [PubMed]

18. Feng, C.; Dai, S.; Wang, L. Optical aptasensors for quantitative detection of small biomolecules: A review. Biosens. Bioelectron. 2014, 59, 64-74. [CrossRef] [PubMed]

19. Armani, A.M.; Kulkarni, R.P.; Fraser, S.E.; Flagan, R.C.; Vahala, K.J. Label-free, single-molecule detection with optical microcavities. Science 2007, 317, 783-787. [CrossRef] [PubMed]

20. Berneschi, S.; Farnesi, D.; Cosi, F.; Conti, G.N.; Pelli, S.; Righini, G.C.; Soria, S. High Q silica microbubble resonators fabricated by arc discharge. Opt. Lett. 2011, 36, 3521-3523. [CrossRef] [PubMed]

21. Cosci, A.; Quercioli, F.; Farnesi, D.; Berneschi, S.; Giannetti, A.; Cosi, F.; Barucci, A.; Conti, G.N.; Righini, G.; Pelli, S. Confocal reflectance microscopy for determination of microbubble resonator thickness. Opt. Express 2015, 23, 16693-16701. [CrossRef] [PubMed]

22. Farnesi, D.; Barucci, A.; Righini, G.C.; Conti, G.N.; Soria, S. Generation of hyper-parametric oscillations in silica microbubbles. Opt. Lett. 2015, 40, 4508-4511. [CrossRef] [PubMed]

23. Wang, H.T.; Wu, X. Optical manipulation in optofluidic microbubble resonators. Sci. China Phys. Mech. Astron. 2015, 58, 114206. [CrossRef]

24. Zhang, X.; Liu, T.; Jiang, J.; Liu, K.; Yu, Z.; Chen, W.; Liu, W. Micro-bubble-based wavelength division multiplex optical fluidic sensing. In Proceedings of the International Society for Optics and Photonics, Beijing, China, 9-11 October 2014.

25. Tang, T.; Wu, X.; Liu, L.; Xu, L. Packaged optofluidic microbubble resonators for optical sensing. Appl. Opt. 2016, 55, 395-399. [CrossRef] [PubMed]

26. Hu, H.; White, I.M.; Suter, J.D.; Dale, P.S.; Fan, X. Analysis of biomolecule detection with optofluidic ring resonator sensors. Opt. Express 2007, 15, 9139-9146.

27. Srisa-Art, M.; Dyson, E.C.; de Mello, A.J.; Edel, J.B. Monitoring of real-time streptavidin—Biotin binding kinetics using droplet microfluidics. Anal. Chem. 2008, 80, 7063-7067. [CrossRef] [PubMed]

28. Jung, L.S.; Nelson, K.E.; Stayton, P.S.; Campbell, C.T. Binding and dissociation kinetics of wild-type and mutant streptavidins on mixed biotin-containing alkylthiolate monolayers. Langmuir 2000, 16, 9421-9432. [CrossRef]

29. Zhu, C.; Zhu, X.; Landry, J.P.; Cui, Z.; Li, Q.; Dang, Y.; Mi, L.; Zheng, F.; Fei, Y. Developing an efficient and general strategy for immobilization of small molecules onto microarrays using isocyanate chemistry. Sensors 2016, 16, 378. [CrossRef] [PubMed]

(c) 2018 by the authors. Licensee MDPI, Basel, Switzerland. This article is an open access article distributed under the terms and conditions of the Creative Commons Attribution (CC BY) license (http:/ / creativecommons.org/licenses/by/4.0/). 